Title : will be set by the publisher

Editors : will be set by the publisher

EAS Publications Series, Vol. ?, 2021

\title{
DYNAMICAL ARCHITECTURES OF PLANETARY SYSTEMS INDUCED BY ORBITAL MIGRATION
}

\author{
E. Szuszkiewicz ${ }^{1}$ and J. C. B. Papaloizou ${ }^{2}$
}

\begin{abstract}
The aim of this talk is to present the most recent advances in establishing plausible planetary system architectures determined by the gravitational tidal interactions between the planets and the disc in which they are embedded during the early epoch of planetary system formation. We concentrate on a very well defined and intensively studied process of the disc-planet interaction leading to the planet migration. We focus on the dynamics of the systems in which low-mass planets are present. Particular attention is devoted to investigation of the role of resonant configurations. Our studies, apart from being complementary to the fast progress occurring just now in observing the whole variety of planetary systems and uncovering their structure and origin, can also constitute a valuable contribution in support of the missions planned to enhance the number of detected multiple systems.
\end{abstract}

\section{Diversity of planetary systems and migration-induced resonances}

Multiple-planet systems are of particular interest to test theoretical models of planet formation and the evolution of planetary systems. Just a few days before this conference, Jason Wright (Wright et al. 2009) sent a paper in which he and his colleagues generated a catalogue of the 27 published multiple-planet systems around stars within $200 \mathrm{pc}$ from the Sun to the other participants. The authors made several interesting observations about the systems' statistical properties. At least five of these systems harbour planets which are in or near mean-motion resonance. This fact makes the importance of orbital migration even stronger than immediately after the discovery of giant planets very close to their host stars (e.g. Kley 2000, Papaloizou et al. 2007). These arguments, together with the increasing number of known planets with masses in the range of a few Earth masses, have stimulated our study of migration-induced resonances (e.g. Papaloizou \&

\footnotetext{
${ }^{1} \mathrm{CASA}^{*}$ and Institute of Physics, University of Szczecin, Wielkopolska 15, 70-451 Poland

2 DAMTP, University of Cambridge, Wilberforce Road, Cambridge CB3 0WA, UK
} 
Title : will be set by the publisher

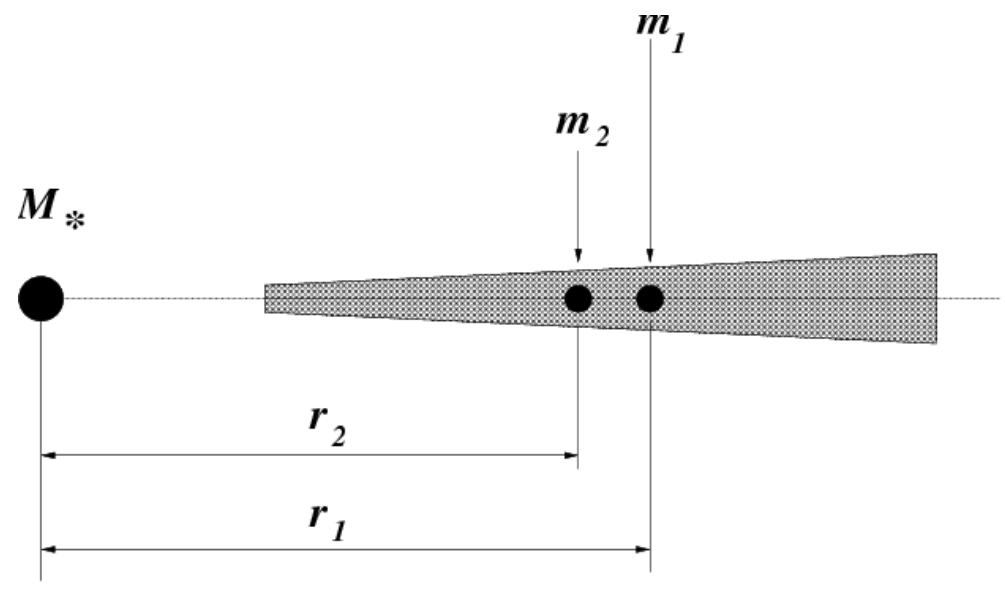

Fig. 1. The initial configuration: two planets, with masses $m_{1}$ and $m_{2}$ respectively, in circular orbits around a central star with mass $M_{*}$ at distances $r_{1}$ and $r_{2}$, are embedded in a gaseous disc.

Szuszkiewicz 2005, Podlewska \& Szuszkiewicz 2008) in systems containing lowmass planets.

Our present goal is to investigate migration-induced architectures of planetary systems, focusing mainly on resonant configurations. In order to achieve this goal we have performed a series of numerical simulations of planets migrating in resonance. The main tools used in our studies are two-dimensional hydrodynamic simulations, simple analytic modelling and N-body investigations. By constructing a simple analytic model, we were able to verify the reliability of our numerical calculations. By combining the hydrodynamic simulations with the N-body technique we were able to follow the dynamical evolution of the planets for a substantial amount of time, comparable with the estimated lifetimes of gaseous discs.

\section{Initial configuration and computational set-up}

Our initial set-up shown in Fig. 1 includes the central star with a mass $M_{*}$ and two orbiting planets with masses $m_{1}$ and $m_{2}$ respectively. The two planets are embedded in a disc which is the source of planet orbit migration. They are initialised on circular orbits around a central mass which has a fixed value of 1 solar mass. The gravitational potential was softened with softening parameter $b=0.8 H$, where $H$ is the semi-thickness of the disc. This results in the formation of an equilibrium atmosphere around the embedded planet which then does not accrete. This softening also allows an adequate representation of type I migration in two dimensional discs (see e.g. Nelson \& Papaloizou 2004). The disc in which planets are initially embedded has a surface density profile $\Sigma(r)$ essentially flat (see Papaloizou \& Szuszkiewicz 2005] for a full description). The planets are located in the flat 
part of this distribution. We use four different values for the maximum value of the surface density $\Sigma_{0}$, namely the standard value of $2 \times 10^{3} \mathrm{~kg} / \mathrm{m}^{2}$, attributed to the minimum mass solar nebula at $5.2 \mathrm{AU}$ which we denote $\Sigma_{1}$, then $\Sigma_{0.5}=0.5 \Sigma_{1}$, $\Sigma_{2}=2 \Sigma_{1}$ and finally $\Sigma_{4}=4 \Sigma_{1}$. The radial boundaries were taken to be open.

The calculations presented here were performed with a two-dimensional version of the Eulerian hydrodynamic code NIRVANA. For details of the numerical scheme and code adopted, see Nelson et al. (2000).

\section{A Super-Earth $\left(m_{1}=4 M_{\oplus}\right)$ and an Earth analogue $\left(m_{2}=1 M_{\oplus}\right)$}

Let us consider here a system of two planets with disparate masses, namely a Super-Earth with a mass of $4 \mathrm{M}_{\oplus}$ and an Earth analogue $\left(1 \mathrm{M}_{\oplus}\right)$. The time-scale of inward migration for a low-mass planet on a circular orbit embedded in a disc with constant surface density can be approximated by the formula given by Tanaka et al. (2002)

$$
\tau_{r}=\left|\frac{r_{p}}{\dot{r}_{p}}\right|=W_{m} \frac{M_{*}}{m_{p}} \frac{M_{*}}{\Sigma_{p} r_{p}^{2}}\left(\frac{c}{r_{p} \Omega_{p}}\right)^{2} \Omega_{p}^{-1}
$$

Here $M_{*}$ is the mass of the central star, $m_{p}$ is the mass of the planet orbiting at distance $r=r_{p}, \Sigma_{p}$ is the disc surface density at $r=r_{p}, c$ is the local sound speed and $\Omega_{p}$ is the angular velocity at $r=r_{p}$. The numerical coefficient $W_{m}$ is 0.3704 .

The density waves excited by a low mass planet with small eccentricity in the disc lead to orbital circularization (e.g. Artymowicz 1993, Papaloizou \& Larwood 2000) at a rate that can be estimated as (Tanaka \& Ward 2004)

$$
t_{c}=\frac{\tau_{r}}{W_{c}}\left(\frac{c}{r_{p} \Omega_{p}}\right)^{2} .
$$

Here, the numerical coefficient $W_{c}$ is equal to 0.289 .

It is expected from equation (3.1) that two planets with different masses will migrate at different rates. In our case, the Super-Earth in the external orbit will migrate faster than the Earth analogue. This has the consequence that their period ratio will evolve with time and may accordingly attain and become locked in a mean-motion resonance (Nelson \& Papaloizou 2002, Kley et al. 2000). We have considered the pair of planets to be evolving in discs with $\Sigma_{0}=\Sigma_{0.5}, \Sigma_{0}=$ $\Sigma_{1}, \Sigma_{0}=\Sigma_{2}$ and $\Sigma_{0}=\Sigma_{4}$. The evolution was followed until a resonance was established. The results are summarised in Fig. 2 (left panel) where the evolution of the ratio of the semi-major axes, which starts from the value 1.2 for all four cases, is shown. The fastest migration (steepest slope) corresponds to the case where the two planets are embedded in a disc with $\Sigma_{0}=\Sigma_{4}$ and the slowest to the case of a disc with $\Sigma_{0}=\Sigma_{0.5}$. The planets in the disc with $\Sigma=\Sigma_{4}$ become trapped in a $p+1: p=8: 7$ resonance. If the disc surface density is two times smaller, then a 7:6 resonance is attained. If it is four or eight times smaller, then the attained resonance is 5:4. These results are fully consistent with the idea that higher $p$ resonances are associated with faster relative migration rates. Let us concentrate 
Title : will be set by the publisher
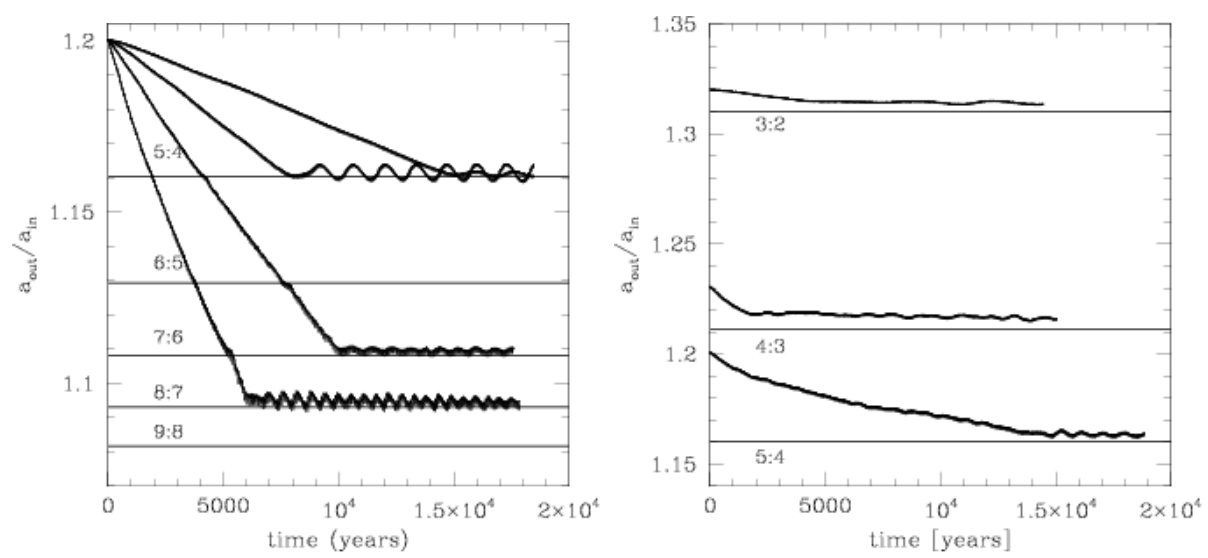

Fig. 2. (left) The evolution of the ratio of semi-major axes for the two planets with masses $m_{1}=4 M_{\oplus}$ and $m_{2}=1 M_{\oplus}$. Starting from the lower curve and going upwards, the curves correspond to the initial surface density scalings $\Sigma_{0}=\Sigma_{4}, \Sigma_{0}=\Sigma_{2}, \Sigma_{0}=\Sigma_{1}$, and $\Sigma_{0}=\Sigma_{0.5}$ respectively. (right) The semi-major axis ratio when $m_{1}=m_{2}=4 M_{\oplus}$ with $\Sigma_{0}=\Sigma_{1}$ (uppermost curve) and with $\Sigma_{0}=\Sigma_{4}$ (two lower curves)

on the 8:7 resonance, illustrating our combined methodology of hydrodynamic calculations (an explicit evolution of a 2D gaseous disc with embedded planets is calculated), simple analytic modelling and N-body techniques (the migration and eccentricity damping due to the gaseous disc is included in the form of analytic prescriptions).

\subsection{Two-dimensional hydrodynamic simulations}

The whole evolution for the fastest migration rate is illustrated in Fig. 3. This shows the evolution of the individual semi-major axes, eccentricities, angle between apsidal lines and one of the resonant angles. The inner planet did not migrate significantly until an 8:7 resonance was attained at about 6000 years. Subsequently, the two planets migrated inwards together, maintaining the commensurability. During 18000 years of evolution the outer planet changed its location from a dimensionless radius of 1.2 to 0.9 . The eccentricity of the outer planet increased slightly and that of the inner planet substantially, reaching an equilibrium value of 0.04 at around 10000 years and at later times oscillating around this value. At the end of the simulation shown here, the ratio of eccentricities $e_{1} / e_{2}$ is equal to 0.125 . The local peaks in the values of inner planet eccentricity occurring at 2000, 4000 and around 5400 years correspond to the planet passing through the 5:4, 6:5 and 7:6 resonances respectively. After about 8000 years the angle between the apsidal lines oscillates around $212^{\circ}$ and the resonant angle $\phi$ around $264^{\circ}$. The amplitude of the oscillations for both angles decreases with time. 

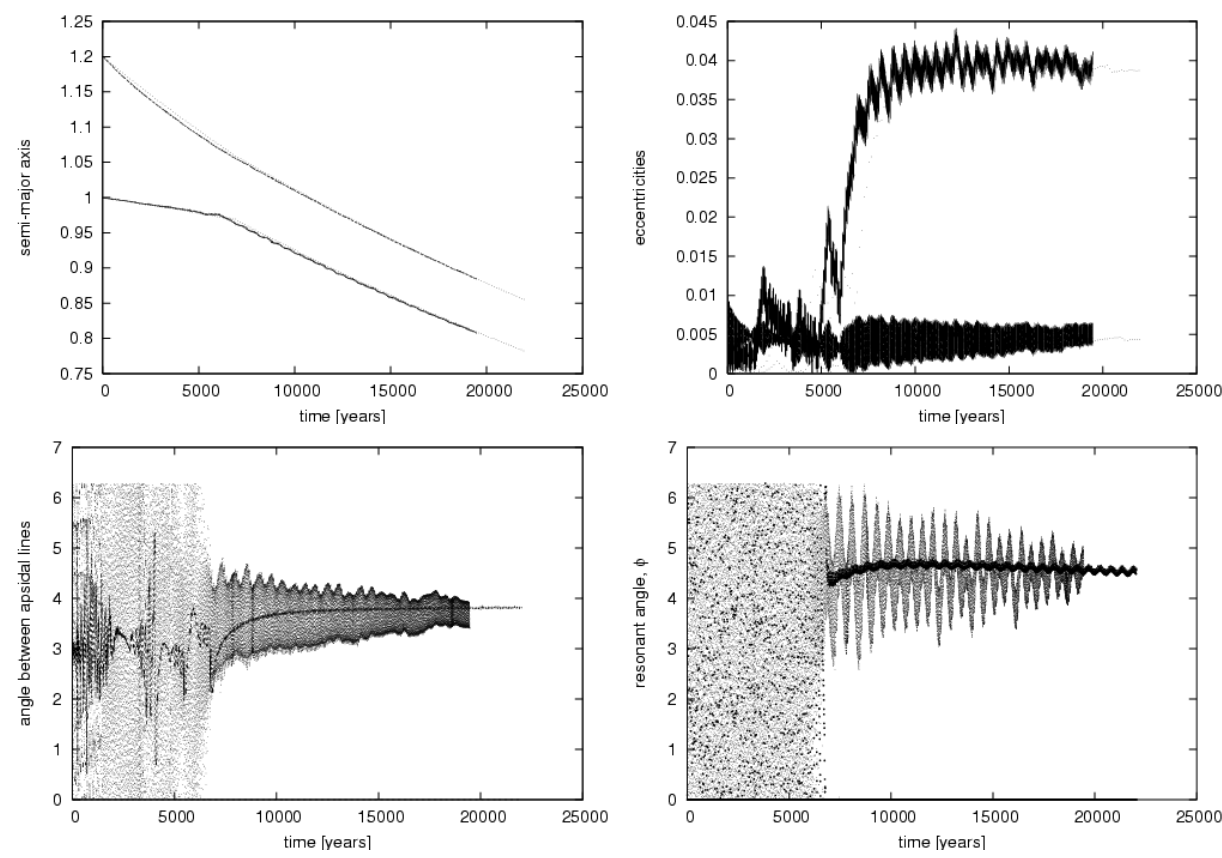

Fig. 3. The evolution of semi-major axes, eccentricities, angle between apsidal lines and resonant angle for two planets with masses, $m_{1}=4 M_{\oplus}$ and $m_{2}=1 M_{\oplus}$ migrating towards a central star embedded in the disc with $\Sigma=\Sigma_{4}$, obtained by hydrodynamic simulations and N-body simulations (dotted lines in two upper panels and dark lines in two lower panels).

\subsection{Comparison with the analytic model}

It is of interest to compare the eccentricities obtained above when the planets are trapped in a commensurability with what is expected when resonant effects and disc tides are in balance. Papaloizou \& Szuszkiewicz (2005) have given a simple approximate analytic solution for two migrating planets locked in a $p+1: p$ commensurability. In particular, they have shown that if eccentricities are not too large, then when they stop growing, they must satisfy

$$
\frac{e_{1}^{2}}{t_{c 1}}+\frac{e_{2}^{2}}{t_{c 2}} \frac{m_{2} n_{1} a_{1}}{m_{1} n_{2} a_{2}}-\left(\frac{e_{1}^{2}}{t_{c 1}}-\frac{e_{2}^{2}}{t_{c 2}}\right) f=\left(\frac{1}{t_{m i g 1}}-\frac{1}{t_{m i g 2}}\right) \frac{f}{3},
$$

where $f=m_{2} a_{1} /\left((p+1)\left(m_{2} a_{1}+m_{1} a_{2}\right)\right)$. Here the semi-major axes and eccentricities of the two planets $(i=1,2)$ are $a_{i}$ and $e_{i}$. The migration rates (assumed directed inwards) and circularization times induced by the disc tides are $t_{m i g i}=\left|n_{i} / \dot{n}_{i}\right|=\left|2 a_{i} /\left(3 \dot{a}_{i}\right)\right|=2 \tau_{r} / 3$, and $t_{c i}=\left|e_{i} / \dot{e}_{i}\right|$ respectively. The mean motions are $n_{i}$.

In the simulations presented here $e_{2}>>e_{1}$. We can simplify matters even further, setting $e_{1}=0$. We can also assume that $p$ is large, because in the present 
example its value is 7 . We also note that from equations $(3.1)$ and $(3.2)$ it turns out that $t_{c}=\left(\tau_{r} / W_{c}\right)(H / r)^{2}$. Thus we obtain

$$
e_{2}^{2}=\left(\frac{m_{1}}{m_{2}}\left(\frac{a_{2}}{a_{1}}\right)^{1 / 2}-1\right) \frac{m_{1}}{0.578(p+1)\left(m_{2}+m_{1}\right)}(H / r)^{2} .
$$

If we apply the above relation to the case illustrated in Fig. 3 for the two planets with masses $m_{1}=4 M_{\oplus}$ and $m_{2}=M_{\oplus}$ embedded in a disc with the aspect ratio $H / r=0.05$ and initial surface density scaling $\Sigma_{0}=\Sigma_{4}$, we obtain $e_{2}=0.037$ in reasonable agreement with the simulations.

\subsection{Comparison between hydrodynamic simulations and $\mathrm{N}$-body calculations}

The hydrodynamic calculations discussed in the previous sections allow us to follow the migrations of planets for almost $2 \times 10^{4}$ years. As a result, we have been able to simulate planets becoming trapped in resonances. The behaviour and stability of the resonance trapping vary with the planet masses and the surface density of the disc in which they are embedded. The outcomes of these simulations could be well matched to those of N-body integrations where we incorporate simple prescriptions for the migration and eccentricity damping given through equations $3.1,3.2$. Using these expressions in the N-body code we have extended the hydrodynamic calculations for a longer period of time and studied the long term stability of the resonances that we found. As a first step we have adjusted the numerical coefficients in equations 3.1 3.2 in such a way that the hydrodynamic and Nbody approaches give the same qualitative evolution.

As an example, in Fig. 3 we show the results of the comparison for the case of two planets with masses $1 M_{\oplus}$ and $4 M_{\oplus}$ respectively embedded in a disc with initial surface density scaling parameter $\Sigma_{0}=\Sigma_{4}$. The numerical coefficients adopted were $W_{m}=0.3647$ and $W_{c}=0.225$. These results show good agreement with the hydrodynamic results and display the same 8:7 commensurability. The fitted coefficients were also reasonably close to those expected from analytic disc-planet interaction theory.

The evolution was followed using the N-body approach for an additional $1.2 \times 10^{6}$ years. The two planets remain in the 8:7 resonance during this time and there is no indication of any significant change in the monitored quantities for the last $9 \times 10^{5}$ years. The long term evolution of this system is shown in Fig. 4 . It is interesting to note that the equilibrium value of eccentricity of the inner planet is around $e_{2}=0.03$, in accordance with the value predicted by the simple analytic model discussed in Section 3.2 .

\section{Two Super-Earths $\left(m_{1}=m_{2}=4 M_{\oplus}\right)$}

The resonant interaction must balance the tendency towards relative migration of the two planets. This is smaller when the planets have the same mass. In order to investigate this fact, two planets of mass $4 M_{\oplus}$ were initiated close to a $3: 2$ 

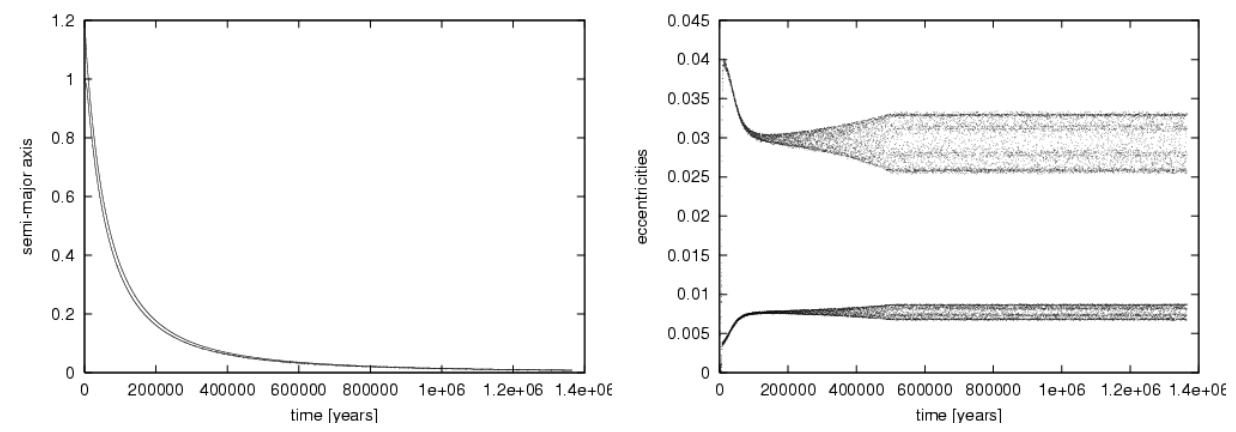

Fig. 4. The evolution of the semi-major axes and eccentricities for two planets with masses $m_{1}=4 M_{\oplus}$ and $m_{2}=1 M_{\oplus}$ migrating towards a central star embedded in the disc with $\Sigma=\Sigma_{4}$ obtained from N-body simulations.

resonance with $r_{1}=1.32$ and $r_{2}=1$ in a disc with $\Sigma_{0}=\Sigma_{1}$. The evolution of the semi-major axis ratio for the planet orbits is shown in Fig. 2 (right panel). We also performed simulations in a disc with $\Sigma_{0}=\Sigma_{4}$ for the same masses starting at $r_{1}=1.23$ and $r_{2}=1.00$ and at $r_{1}=1.20, r_{2}=1.00$. The planets become trapped in the nearest available resonance which is a good indication of stability. The evolution of the equal mass pair of planets is discussed more extensively in Papaloizou \& Szuszkiewicz (2005) and in Eacny \& Szuszkiewicz (this volume).

\section{A gas giant and a Super-Earth $\left(m_{1}=333 M_{\oplus}, m_{2}=5.5 M_{\oplus}\right)$}

In the previous sections we have investigated systems in which both planets have a low mass. Here we extend our study to the case where one planet is a Super-Earth and the other a gas giant. The migration rates for different planet masses has been estimated by a number of authors, see the review by Papaloizou et al. (2007). Their results are illustrated in Fig. 5 (left panel), where we plot the migration time of a planet as a function of its mass. There are two mass regimes which are of interest here, namely $\left(0.1-30 M_{\oplus}\right)$ and $\left(150-1500 M_{\oplus}\right)$, for which in a typical protoplanetary disc we can talk about two different types of migration, called type I and type II respectively. The migration time for low mass planets embedded in a gaseous disc (type I migration) has been discussed above in Section 3. Type II migrators open a gap in the disc and their evolution is determined by the radial velocity drift in the disc $v_{r}$. The migration time can be estimated as follows (Lin and Papaloizou 1993)

$$
\tau_{I I}=\frac{r_{p}}{v_{r}}=\frac{2 r_{p}^{2}}{3 \nu}
$$

where $\nu$ is a kinematic viscosity parameter. This has been shown in Fig. 5 (left panel) for $r_{p}=5.2 \mathrm{AU}$ and different values of $\nu\left(10^{-5}, 2 \times 10^{-5}, 3 \times 10^{-5}, \ldots, 9\right.$ $\times 10^{-5}$ and $10^{-6}$ ) expressed in dimensionless units. In drawing lines for a given 

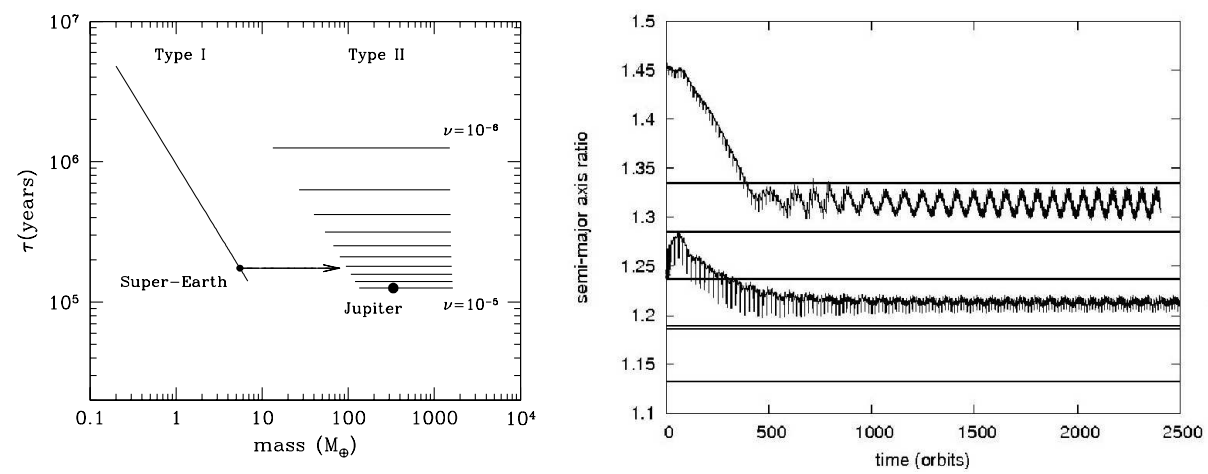

Fig. 5. (left) Comparison between the times of migration for planets with different masses located at 5.2 AU, embedded in a disc with a flat surface density distribution, the standard value attributed to the minimum mass solar nebula at $5.2 \mathrm{AU}$, namely $\Sigma=2000$ $\mathrm{kg} / \mathrm{m}^{2}$ and $\mathrm{H} / \mathrm{r}=0.05$. (right) The evolution of the ratio of semi-major axes for the Jupiter and Super-Earth embedded in a gaseous disc. In the case of the upper curve the initial semi-major axis ratio is 1.45 and planets became locked into a 3:2 resonance. For the lower curve, the initial semi-major axis ratio is 1.24 and the attained commensurability is 4:3. The solid horizontal lines are the 3:2, 4:3 and 5:4 resonance widths in the case of circular orbits in the restricted three body problem (Wisdom, 1980, Lecar et al. 2001).

viscosity parameter we have taken into account the condition for a gap opening in the disc which reads $\frac{m_{p}}{M_{*}}>\frac{40 \nu}{r_{p}^{2} \Omega_{p}}$. From this expression, it is clear that the bigger the viscosity $\nu$, the bigger the mass of the planet must be to be able to open a gap. We have chosen the mass of the type I and type II migrators to be $5.5 M_{\oplus}$ and one Jupiter mass respectively. Their locations have been marked in Fig. 5 according to their masses and, in the case of the Jupiter, also according to the viscosity adopted in the disc $\left(\nu=10^{-5}\right)$. It is obvious from this figure that we should expect a convergent migration if the Jovian type planet is in the external orbit and the Super-Earth in the internal one. Starting from the configuration illustrated in Fig. 5 (left panel), we have studied the possible resonances in this system. In order to investigate how the outcome of such evolution depends on the initial planet configurations, we have performed our simulations with a wide range of planetary separations (see Table 1 in Podlewska \& Szuszkiewicz 2008). The 2:1 commensurability was not taken into account because of computational time constraints. The resonances located closer to the Jupiter, such as 5:4, 6:5 or higher values of $p$, are not possible, because for such small separations the system becomes unstable and the Super-Earth is scattered from the disc. We have found that two outcomes are possible, namely either the Super-Earth is ejected from the disc or the planets become locked in 3:2 or 4:3 mean-motion resonances. In Fig. 5 (right panel) we show two examples of resonant trappings occurring when 
the relative planet separation is 0.45 (upper curve) and 0.24 (lower curve). In the first case, the differential migration brought planets into a 3:2 commensurability and in the second case into a 4:3. The semi-major axis ratio of planets librates exactly within the regions of the resonance width marked in Fig. 5 (right panel) by solid, horizontal lines. Both commensurabilities shown in Fig. 5 are stable for the whole time of our simulations, namely 2500 orbits (the unit of time is defined as the orbital period on the initial orbit of the Super-Earth divided by $2 \pi)$. For full discussion of these results see Podlewska \& Szuszkiewicz (2008) and also Podlewska (this volume).

\section{Conclusions}

Particular attention has been devoted here to the investigation of the occurrence of resonant configurations in systems containing low-mass planets. Studies of commensurabilities have already been applied successfully to analyse the motion of pulsar planets and they proved to be a powerful tool in that context. They have the potential to be similarly useful in our search for Earth-like planets in other systems. Our prediction of the occurrence of the commensurabilities in such systems may turn out to be particularly valuable in the case of the TTV (Transit Timing Variation) observations, because the differences in the time intervals between successive transits, caused by planet-planet interactions, are largest near meanmotion resonances (e.g. Agol et al. 2005). Finally, detection of such resonances can also yield useful information about orbital migration as a process operating during planet formation.

\section{Acknowledgements}

This work has been partially supported by MNiSW grant N203 026 32/3831 (20072010), the project ASTROSIM-PL and KITP, Santa Barbara, NSF Grant No PHY99-0794. E.S. would like to express her gratitude for support through a PPARC funded visitor's grant and for the provision of computer facilities at the Astronomy Unit, Queen Mary, University of London. The simulations reported here were also performed using the Polish National Cluster of Linux Systems (CLUSTERIX) and the computational cluster HAL9000 of the Faculty of Mathematics and Physics at the University of Szczecin.

\section{References}

Agol, E., Steffen, J., Sari, R., Clarkson, W., 2005, MNRAS, 359, 567

Artymowicz, P., 1993, ApJ, 419, 155

Kley, W., 2000, MNRAS, 313, L47

Kley, W., Peitz, J., Bryden, G., 2004, A\&A, 414, 735

Lecar, M., Franklin, F. A., Holman, M. J., Murray, N. J., 2001, ARA\&A 39, 581 
Lin D. N. C., Papaloizou J. C. B., 1993, in Protostars \& Planets III, eds. E. H. Levy, \& J. I. Lunine (Tucson: Univ. Arizona Press), 749

Nelson, R. P., Papaloizou, J. C. B., Masset, F., Kley, W., 2000, MNRAS 318, 18

Nelson R. P. and Papaloizou, J. C. B., 2002, MNRAS, 333, 26

Nelson R. P. and Papaloizou, J. C. B., 2004, MNRAS, 350, 849

Papaloizou, J.C.B., Larwood, J.D., 2000, MNRAS, 315, 823

Papaloizou, J. C. B., Szuszkiewicz, E., 2005, MNRAS, 363, 153

Papaloizou, J. C. B., Nelson, R. P., Kley, W., Masset, F., Artymowicz, P., 2007, in Protostars \& Planets V, Reipurth, D. Jewitt, \& K. Keil (eds.), University of Arizona Press, Tucson, 2007

Podlewska, E., Szuszkiewicz, E., 2008, MNRAS, 386, 1347

Tanaka, H., Ward, W.R., 2004, ApJ, 602, 388

Tanaka H., Takeuchi, T., Ward, W. R., 2002, ApJ, 565, 1257

Wisdom, J., 1980, AJ, 85, 1122

Wright, J. T., Upadhyay, S., Marcy, G. W., Fischer, D. A., Ford, E. B., Johnson, J. A., 2009, ApJ, 693, 1084 